Gut, 1986, 27, 1014-1017

\title{
Bladder smooth muscle dysfunction in patients with irritable bowel syndrome
}

\author{
P J WHORWELL, E W LUPTON, D ERDURAN, AND K WILSON \\ From the Departments of Medicine and Urology, University Hospital of South Manchester, Manchester
}

SUMMARY Urodynamic studies were carried out on 30 patients with irritable bowel syndrome and 30 matched controls. Fifty per cent of the irritable bowel patients compared with only $13 \%$ of the control group had evidence of bladder dysfunction $(p=0.006)$. In the irritable bowel group detrusor instability was observed in 10 patients compared with only one control subject $(p=0 \cdot 008)$. A steep cystometrogram occurred in five irritable bowel patients and three controls (NS). Detrusor instability was most common in patients with a bowel habit characterised by alternating constipation and diarrhoea. This is the first study to provide objective evidence that patients with irritable bowel syndrome may have a disorder of smooth muscle or its innervation that is not confined to the gastrointestinal system.

Although the pathogenesis of irritable bowel syndrome is uncertain it seems likely that a disturbance of gastrointestinal smooth muscle function is contributory. ${ }^{1-5}$ It was originally considered that this abnormality was confined to the colon, but subsequent studies have shown both oesophageal ${ }^{6}$ and small intestinal involvement. ${ }^{7}$ We have recently reported that compared with healthy controls patients with irritable bowel syndrome complain of a significant number of urinary symptoms such as frequency, nocturia, and urgency. ${ }^{8}$ It is not known, however, whether this reflects an intrinsic abnormality of bladder smooth muscle or is related to extrinsic compression associated with the gut problem.

Detrusor instability is a well recognised disturbance of vesical function and may give rise to frequency, nocturia, and urgency. It may have obstructive neuropathic, or idiopathic aetiologies and is defined as the occurrence of detrusor contractions which the patient is unable to inhibit. ${ }^{9}$ Symptoms such as frequency and urgency may also be associated with a steep cystometrogram which is an indication of indistensibility of the bladder wall. ${ }^{10}$ to ascertain whether these or other abnormalities of bladder function account for the urinary symptoms

Address for correspondence: Dr P J Whorwell, Dept of Medicine, University Hospital of South Manchester, Nell Lane, West Didsbury, Manchester M20 8LR.

Received for publication 3 January 1986 associated with irritable bowel syndrome, urodynamic studies were done in a group of patients with and without this disorder.

\section{Methods}

PATIENTS

Of 44 consecutive female patients (age range 25-65 years, mean age 43) with irritable bowel syndrome attending the outpatient department 30 agreed to participate in the study. Four of the 14 patients declining investigation did not have urinary symptoms compared with six of the 30 in the study group. This difference was not significant. The diagnosis of irritable bowel syndrome was based on the presence of abdominal pain, abdominal distension, and a disturbed bowel habit (diarrhoea three, constipation 17, alternating diarrhoea and constipation 10). All subjects had normal biochemistry, haematology and colonoscopy or contrast radiology. Because of the small risk of infection ethical approval to do urodynamic measurements on normal control subjects was not obtained. It was therefore decided to study as a matched control group 30 consecutive female patients (age range 20-66 years, mean age 46) attending a urology clinic complaining of urinary frequency in whom the diagnosis of irritable bowel syndrome had been excluded by the absence of the symptoms listed above. All subjects had normal midstream urinary specimens. The psychological 
status of all patients was assessed using the 28 question general health questionnaire. ${ }^{11}$

All patients completed frequency volume charts to allow an objective assessment of their voiding patterns. All medication was discontinued for 24 hours before urodynamic studies were carried out using a technique similar to that described by Bates and Corney ${ }^{12}$ but without radiographic monitoring. During filling cystometry the detrusor pressure was measured with respect to the volume of fluid within the bladder (Figure). Fast filling cystometry (100 $\mathrm{ml} /$ minute) was carried out in both supine and vertical positions until the patient experienced an intense desire to void. Other provocative stimuli were coughing and posture change. Detrusor instability was diagnosed if intrinsic, phasic detrusor contractions with an amplitude in excess of $15 \mathrm{~cm}$ $\mathrm{H}_{2} \mathrm{O}$ were identified. Impaired bladder distensibility was detected by a persistent steep rise in the intrinsic detrusor pressure above $15 \mathrm{~cm} \mathrm{H}_{2} \mathrm{O}$ (steep cystometrogram). Examples of normal steep and unstable cystometrograms are shown in the Figure. After filling cystometry a voiding pressure/flow study was carried out. Group comparisons were done using contingency table analysis $\left(\chi^{2}\right)$.

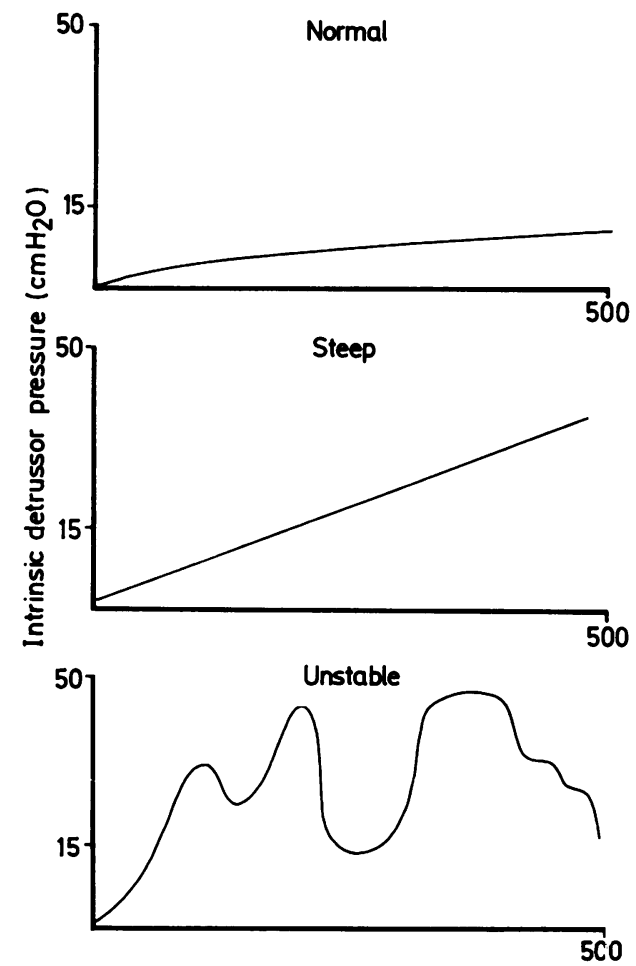

Figure Examples of normal, steep, and unstable cystometrograms. 


\section{Results}

Fifty per cent of the irritable bowel patients compared with only $13 \%$ of the control group had evidence of bladder dysfunction $(p=0.006)$. In the irritable bowel group detrusor instability was observed in 10 patients compared with only one control subject $(p=0 \cdot 008)$. A steep cystometrogram occurred in five irritable bowel patients and three controls (NS). There were no significant voiding abnormalities in patients or controls.

The Table details urodynamic findings, urinary symptoms, and bowel habit in the irritable bowel syndrome patients. When the bladder abnormalities were related to bowel habit there was a tendency for instability to be more common in patients with alternating constipation and diarrhoea and less common in constipated patients. The numbers were, however, too small to be significant. The number of steep cystometrograms was small but their distribution did not appear to be uneven. The psychological score did not correlate with urinary symptomatology or any particular urodynamic abnormality.

Of the urinary symptoms complained of by the irritable bowel syndrome patients urgency and urge incontinence were most likely to predict bladder instability. Six patients had no urinary symptoms; of these five had a stable bladder, and one a steep cystometrogram.

\section{Discussion}

This study clearly shows that a demonstrable bladder abnormality is common in patients with irritable bowel syndrome. It is difficult to assess the incidence of bladder dysfunction in the normal population because of the ethical considerations associated with such studies and the choice of an appropriate control group presents considerable problems. In order to obtain some estimate of the incidence of bladder abnormalities in a non-irritable bowel population a group of patients in whom this diagnosis had been excluded attending a urology clinic complaining of urinary frequency was chosen. By virtue of their urinary symptoms it might be expected that this control group would if anything bias the study away from a positive result. Ten per cent of the control group had an abnormality but only one $(3 \%)$ showed detrusor instability.

It has been estimated that $10 \%$ of the population may have an unstable bladder, ${ }^{13}$ a considerably higher figure than in our control group. This discrepancy may be accounted for by the inclusion of people with irritable bowel syndrome in the population estimate as this is such a common condition, affecting up to $15 \%$ of the com- munity. ${ }^{14} 15$ One explanation for the prominence of urinary symptoms in irritable bowel syndrome could be some form of extrinsic bladder compression possibly related to the abdominal distension which is a predominant feature of this condition. This may account for the steep cystometrograms (implying indistensibility of the bladder wall) observed in some patients. The finding of a high incidence of detrusor instability in irritable bowel syndrome in the study, however, strongly suggests that there is also an intrinsic abnormality of bladder smooth muscle or its innervation in patients with this disorder. These results lend support to the hypothesis that irritable bowel syndrome is a more diffuse disorder of smooth muscle than has been hitherto recognised.

The prominence of a particular symptom may well determine whether a patient with irritable bowel syndrome presents to either a gastrointestinal or urological clinic. Awareness of this situation may lead to the more rational management of such patients and the avoidance of unnecessary investigation. It remains to be seen whether the successful treatment of the gastrointestinal aspects of irritable bowel syndrome will be mirrored by an improvement in urological symptomatology.

\section{References}

1 Thompson WG. The irritable bowel. Gut 1984; 25: 305-20.

2 Ritchie J. The irritable bowel syndrome. Part 2 manometric and cineradiographic studies. Clin Gastroenterol 1977; 6: 622-64.

3 Burns TW. Colonic motility in the irritable bowel syndrome. Arch Intern Med 1980; 140: 247-51.

4 Murney RG, Winship DH. The irritable colon syndrome. Clin Gastroenterol 1982; 11: 563-92.

5 Taylor I, Darby C, Hammond P, Basu P. Is there a myoelectrical abnormality in the irritable colon syndrome. Gut 1978; 19: 391-5.

6 Whorwell PJ, Clouter C, Smith CL. Oesophageal motility in the irritable bowel syndrome. Br Med J 1981; 282: 1101-2.

7 Thompson DG, Laidlow JO, Wingate DL. Abnormal small bowel motility demonstrated by radiotelemetry in a patient with irritable colon. Lancet 1979; 2: 1321-3.

8 Whorwell PJ, McCallum M, Creed FH, Roberts CT. Non-colonic features of irritable bowel syndrome Gut 1986; 27: 37-40.

9 Bates CP. Continence and incontinence. Ann $R$ Coll Surg Engl 1971; 49: 18-35.

10 Abrams P, Feneley R, Torrens M. Urodynamics Berlin/Heidelberg/New York, Springer-Verlag: 1983:69-70.

11 Goldberg DP. The detection of psychiatric illness by questionnaire. Institute of Psychiatry Maudsley Monographs 1972; No 21 
12 Bates CP, Corney CE. Synchronous cine/pressure/flow cystography: a method of routine urodynamic investigation. $\mathrm{Br} J$ Radiol 1971; 44: 44-50.

13 Turner-Warwick R. Observations on the function and dysfunction of the sphincter and detrusor mechanisms. Urol Clin N Am 1979; 6: 13-30.
14 Thompson WG, Heaton KW. Functional bowel disorders in apparently healthy people. Gastroenterology 1980; 79: 283-8.

15 Drossman DA, Sandler RS, McKee DC, Lovitz AJ. Bowel dysfunction among subjects not seeking health care. Gastroenterology 1982; 83: 529-34. 